\title{
TU/e EmonOWEN

\section{Optimal MSE polarisation angle and q-profile estimation using Kalman filters and the plasma simulator RAPTOR}

\section{Citation for published version (APA):}

EUROfusion MST1 Team, ASDEX-Upgrade team, \& Messmer, M. C. C. (2019). Optimal MSE polarisation angle and q-profile estimation using Kalman filters and the plasma simulator RAPTOR. Plasma Physics and Controlled Fusion, 61(3), [035011]. https://doi.org/10.1088/1361-6587/aaf7ed

DOI:

10.1088/1361-6587/aaf7ed

Document status and date:

Published: 01/02/2019

\section{Document Version:}

Publisher's PDF, also known as Version of Record (includes final page, issue and volume numbers)

\section{Please check the document version of this publication:}

- A submitted manuscript is the version of the article upon submission and before peer-review. There can be important differences between the submitted version and the official published version of record. People interested in the research are advised to contact the author for the final version of the publication, or visit the $\mathrm{DOI}$ to the publisher's website.

- The final author version and the galley proof are versions of the publication after peer review.

- The final published version features the final layout of the paper including the volume, issue and page numbers.

Link to publication

\section{General rights}

Copyright and moral rights for the publications made accessible in the public portal are retained by the authors and/or other copyright owners and it is a condition of accessing publications that users recognise and abide by the legal requirements associated with these rights.

- Users may download and print one copy of any publication from the public portal for the purpose of private study or research.

- You may not further distribute the material or use it for any profit-making activity or commercial gain

- You may freely distribute the URL identifying the publication in the public portal.

If the publication is distributed under the terms of Article 25fa of the Dutch Copyright Act, indicated by the "Taverne" license above, please follow below link for the End User Agreement:

www.tue.nl/taverne

Take down policy

If you believe that this document breaches copyright please contact us at:

openaccess@tue.nl

providing details and we will investigate your claim. 
PAPER • OPEN ACCESS

Optimal MSE polarisation angle and q-profile estimation using Kalman filters and the plasma simulator RAPTOR

To cite this article: M C C Messmer et al 2019 Plasma Phys. Control. Fusion 61035011

View the article online for updates and enhancements.

\section{IOP ebooks}

Bringing you innovative digital publishing with leading voices

to create your essential collection of books in STEM research.

Start exploring the collection - download the first chapter of every title for free. 


\title{
Optimal MSE polarisation angle and q-profile estimation using Kalman filters and the plasma simulator RAPTOR
}

\author{
M C C Messmer ${ }^{1}$ (1) , F Felici ${ }^{1}(1)$, O Sauter $^{2}$, A A Teplukhina ${ }^{2}$, J P G Loenen ${ }^{1}$, \\ M Reich $^{3}$, R Fischer ${ }^{3}$, D Rittich ${ }^{3}$, R J E Jaspers ${ }^{1,4}$, the ASDEX Upgrade \\ Team and the EUROfusion MST1 team ${ }^{5}$ \\ ${ }^{1}$ Eindhoven University of Technology (TU/e), $5600 \mathrm{MB}$ Eindhoven, The Netherlands \\ ${ }^{2}$ École Polytechnique Fédérale de Lausanne (EPFL), Swiss Plasma Center (SPC), CH-1015 Lausanne, \\ Switzerland \\ ${ }^{3}$ Max-Planck-Institut für Plasmaphysik, Boltzmannstr. 2, D-85748 Garching, Germany \\ ${ }^{4}$ Department of Applied Physics, Ghent University, B-9000 Ghent, Belgium \\ E-mail: m.c.c.messmer@tue.nl
}

Received 17 October 2018, revised 28 November 2018

Accepted for publication 11 December 2018

Published 1 February 2019

\begin{abstract}
Accurate $q$-profile reconstruction is of importance for the development of advanced scenarios, but continues to be a challenge in tokamak research. To constrain the $q$-profile in the plasma centre the motional Stark effect diagnostic (MSE) is often used, however achieving routine measurements with the required accuracy proves to be difficult in many devices. We present a novel approach to obtain accurate estimates of the $q$-profile using an observer based approach. The observer combines MSE measurements with a model-based prediction of the system. For this the plasma transport simulator RAPTOR is coupled with a fixed-boundary equilibrium solver to create a model-based prediction of the MSE measurements. An extended Kalman filter is used to merge profile evolution predictions from the RAPTOR code with measurements. Using synthetic data we demonstrate accurate $q$-profile estimations in situations where the model is purposely disturbed and only erroneous MSE measurements are available. For shots at ASDEX Upgrade we show that by constraining RAPTOR with MSE measurements, the evolution of the model's $q$-profile is in close proximity to reference profiles of reconstructed equilibria from an integrated diagnostic suite.
\end{abstract}

Keywords: MSE, RAPTOR, $q$-profile reconstruction, observer, extended Kalman filter, tokamak control, motional Stark effect

(Some figures may appear in colour only in the online journal)

\section{Introduction}

Of the available concepts for nuclear fusion reactors, the most matured one is the tokamak. It confines the plasma by a helical magnetic field which is created by external coils and a current running through the plasma. The externally applied toroidal component of the magnetic field is known, however the field components generated by the plasma current are

5 See the author list of H Meyer et al 2017 Nucl. Fusion 57102014. difficult to measure and manipulate. Tailoring the plasma's current density profile $J$, or its derived quantity, the safety factor $q=\mathrm{d} \Phi / \mathrm{d} \psi$, with toroidal magnetic flux $\Phi$ and poloidal magnetic flux $\psi$, is essential for plasma stability and performance [1].

Unfortunately, no direct measurement of $q$ (or $J$ ) is available. The plasma equilibrium, specifically the distribution of poloidal flux $\psi(R, Z)$ and the free functions $p^{\prime}(\psi)$, $F F^{\prime}(\psi)$, are estimated using (real-time) equilibrium reconstruction codes that solve the 2D Grad-Shafranov equation, 
a two-dimensional, nonlinear, elliptic partial differential equation describing the force balance in the plasma [2, 3]. This process usually involves parametrising the internal profiles and solving a least-square problem constrained by internal and external measurements of the plasma. External measurements are magnetic probes, flux loops and Rogowski coils, internal measurements are for example temperature and density measurements. By using magnetic probes, the plasma equilibrium can be reconstructed with good accuracy at the plasma boundary, however in order to constrain the internal current distribution, knowledge of the internal magnetic field is required. In the plasma centre, Grad-Shafranov solvers are typically constrained by measurements from the motional Stark effect (MSE) diagnostic [4] or polarimetric measurements [5]. MSE systems locally measure the polarisation angle $\gamma$ of light emitted by externally injected neutral particles, which is aligned with respect to the magnetic field. A continuous challenge of the MSE diagnostic is to record data with sufficient accuracy for a good $q$-profile reconstruction. For a precise reconstruction, for example to accurately resolve the evolution of the central safety factor during sawtooth events, a measurement accuracy of $0.1^{\circ}$ on a millisecond timescale is required [6, 7].

In this article we propose a novel way to use a modelbased observer to obtain accurate estimates of the magnetic pitch angles. This approach results in higher quality estimates than can be achieved by using only measurement or model data and can also complement situations where direct measurements from MSE are not available. The observer combines MSE measurements with model-based prediction of the system for better estimates of the state of the system. This provides:

- a check of the measurement quality (or the simulation quality),

- better estimates of the state of the model by combining the real and predicted measurement,

- a real-time capable filtering solution,

- a constraint on the $q$-profile by the model in cases where MSE data is not available,

- a correction of the model in case of model mismatch.

As a physics model describing the process, we use the poloidal flux diffusion equation and electron transport equation [8], implemented in the RAPTOR code [9].

A recently developed approach, similar to the one described in this paper, is implemented in the IDE code $[10,11]$. In this approach, a Bayesian approach is used to merge diagnostic measurements related to the core current density with a one-step-ahead prediction based on the poloidal flux diffusion equation. Important differences between both approaches are that RAPTOR is capable to run in realtime and the physics model in IDE only includes the poloidal flux diffusion equation, while the kinetic core profiles are obtained from a Bayesian reconstruction using several diagnostics for individual time slices. The approach of combining RAPTOR with an extended Kalman filter (EKF) contains a predictive model for the electron temperature (with future versions including also ion temperature and particle transport equations [12]), allowing the EKF approach to be applied to reconstruct all the core profiles. At the same time, it is important to realise the similarities between the Bayesian approach of IDE and the Kalman filter approach in this paper. Indeed, it can be shown that the Kalman filter can be written as a special case of a Bayesian estimator assuming the noise is Gaussian [13]. The great advantage of Kalman filters above more general Bayesian reconstruction is its speed, which allows real-time implementation. In previous work [14], RAPTOR has been used on TCV to estimate the core electron temperature and $q$-profile, constrained by measurements of core density and temperature. The code is also used at AUG [15], JET [12] and ITER [16].

In this work, we show for the first time that RAPTOR can be constrained by MSE measurements to accurately model the $q$-profile evolution. A prediction of $\gamma$ is not possible from the state of the model (RAPTOR) alone, but requires knowledge of the 2D magnetic field structure. To be able to calculate $\gamma$, RAPTOR has been coupled to a Grad-Shafranov solver (CHEASE [17]) in a self consistent way, which ensures that the geometry-dependent terms that enter the current diffusion equation (CDE) evolve consistently with the 2D equilibrium, and at the same time the equilibrium is correctly constrained by the $q$-profile from the CDE. This coupling allows a fast and accurate calculation of $\gamma$ from the current density profile given by the $\mathrm{CDE}$ in combination with the plasma equilibrium.

In the future we want to couple RAPTOR to a real-time capable equilibrium solver for real-time $q$-profile prediction and control. Then, instead of constraining the GS solver by RAPTOR's $q$-profile, the filtered polarisation angle would be used as a constraint to ensure consistent $q$-profiles between RAPTOR and the equilibrium solver.

The remainder of this article is structured as follows:

Section 2 introduces the EKF, followed by a description of the RAPTOR code and a brief outline of the MSE diagnostic and the difficulties present at the ASDEX Upgrade tokamak. In section 3 the EKF is first tested on a virtual MSE diagnostic, where $\gamma$ is calculated from the model, and lastly the EKF is used to constrain RAPTOR's $q$-profile with MSE measurements from ASDEX Upgrade. The conclusions are presented in section 4 .

\section{Methology}

\subsection{Extended Kalman filter}

To provide the best estimate of the state of a system, an EKF can be used to filter real measurements with model predicted measurements of the system. The EKF, the nonlinear extension of the Kalman filter (which is used for systems described by linear ODE's), has been described in literature extensively [18]. The EKF has the advantage that it not only takes the measurement and model into account, but it is also recursive, fast and can handle asynchronous measurements. Only the main concepts of the EKF algorithm are outlined here, the 


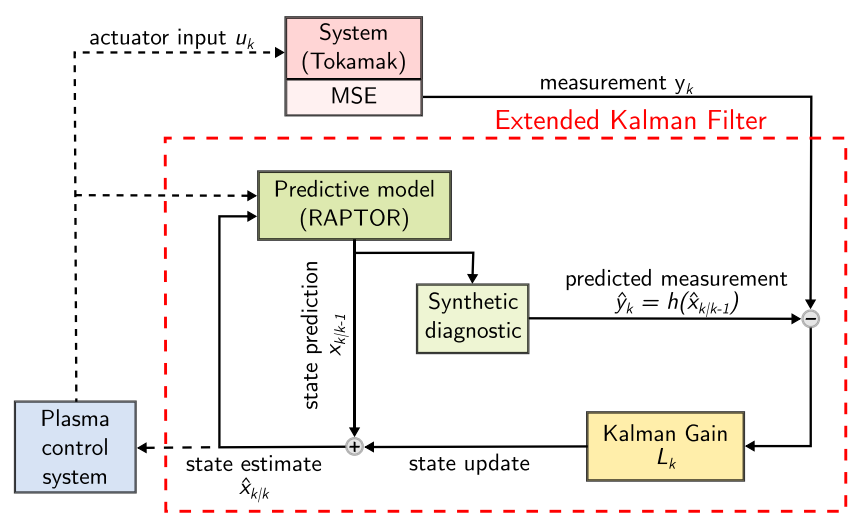

Figure 1. Overview of the extended Kalman filter: The predictive model (RAPTOR) simulates the state of the system from which the measurement (polarisation angles $\gamma$ ) is predicted. In combination with the real measurement the EKF updates the state to obtain an improved estimate.

interested reader is referred to $[18,19]$ for a more detailed description.

The EKF algorithm is illustrated in figure 1. For every time step $k$, the EKF algorithm consists of 4 steps. Our notation follows the one of Simon [18], the hat denotes a predicted quantity from the model and the subset, e.g. $k \mid k-1$, denotes that the quantity is evaluated for time $k$, taking into account the history of the quantity up to time $k-1$

Step 1: The EKF uses a model of the system in the form of a nonlinear ODE $x_{k}=f\left(x_{k-1}, u_{k-1}, w_{k-1}\right)$, where $x_{k}$ describes the state of the system, $u$ are inputs to the model and $w$ is the noise vector of the state. First, the partial derivatives of the model function are calculated

$$
\begin{gathered}
F_{k-1}=\left.\frac{\partial f_{k-1}}{\partial x}\right|_{\hat{x}_{k-1}}, \\
G_{k-1}=\left.\frac{\partial f_{k-1}}{\partial w}\right|_{\hat{x}_{k-1}} .
\end{gathered}
$$

Step 2: Predict the state estimate and estimation-error covariance matrix:

$$
\begin{gathered}
\hat{x}_{k \mid k-1}=f\left(\hat{x}_{k-1}, u_{k-1}, 0\right), \\
\Sigma_{k \mid k-1}=F_{k-1} \Sigma_{k-1} F_{k-1}^{T}+G_{k-1} Q_{k-1} G_{k-1}^{T} .
\end{gathered}
$$

Here, $\Sigma$ is the estimation-error covariance matrix and $Q_{k}$ is the covariance matrix of the model.

Step 3: Calculate the partial derivatives of the predicted measurement function $\hat{y}_{k}=h\left(\hat{x}_{k}, v\right)$ :

$$
\begin{aligned}
& H_{k}=\left.\frac{\partial h_{k}}{\partial x}\right|_{\hat{x}_{k \mid k-1}}, \\
& M_{k}=\left.\frac{\partial h_{k}}{\partial v}\right|_{\hat{x}_{k \mid k-1}},
\end{aligned}
$$

with $v$ being the noise vector of the measurement.
Step 4: Update the state and error covariance estimate:

$$
\begin{aligned}
L_{k} & =\Sigma_{k \mid k-1} H_{k}^{T}\left(H_{k} \Sigma_{k \mid k-1} H_{k}^{T}+M_{k} R_{k} M_{k}^{T}\right)^{-1}, \\
\hat{x}_{k \mid k} & =\hat{x}_{k \mid k-1}+L_{k}\left(y_{k}-h_{k}\left(\hat{x}_{k \mid k-1}\right)\right), \\
\Sigma_{k \mid k} & =\left(I-L_{k} H_{k}\right) \Sigma_{k \mid k-1},
\end{aligned}
$$

where $L_{k}$ is the so called Kalman gain and $y_{k}$ the real measurement of the system.

For a smooth initialisation of the EKF, the Kalman gain is multiplied with a time dependent function:

$$
L_{k}^{\prime}=L_{k} \cdot \exp \left(-0.12 \cdot\left(t-t_{0}\right)\right),
$$

where $t_{0}$ is the first active time step of the observer and the factor 0.12 was chosen to provide a smooth initialisation.

The system is described by the CDE and heat transport equations, implemented by the transport simulator RAPTOR. The state of the model $\hat{x}$ is a representation of the poloidal flux $\psi$ and the electron temperature $T_{e}$, from which various plasma parameters-for this article most importantly the safety factor $q$-can be derived. As measurements $y_{k}$, the polarisation angle measurements from the MSE diagnostic are used. The model, measurement and measurement prediction, will be explained in the following sections.

A central quantity for the EKF algorithm are the noises of the state and measurement, $w_{k}$ and $v_{k}$ respectively, from whose covariance matrices the Kalman Gain $L_{k}$ is calculated (equation (2.7)). The covariance matrices are essential for the performance of the EKF. They determine the weighting of the model, or measurement, and are used to tune the EKF.

The process covariance matrix $Q_{k}$ of the model has previously been defined in [19]. It is designed to enforce a high correlation between neighbouring spatial points based on physical and numerical consideration of the problem.

The covariance matrix of the measurement is a diagonal matrix containing the square of the standard deviation of each measurement channel as diagonal entries. It is described in more detail in section 2.3.

\subsection{Plasma evolution model: RAPTOR}

The observer is based on a model of the system (the tokamak), which provides estimates of the state of the system from which the expected measurement can be derived. The system is modelled by the transport simulator RAPTOR. This faster than real-time capable code solves the coupled 1D poloidal flux $(\psi)$, electron- and ion- temperature $\left(T_{e}, T_{i}\right)$ equations, as well as particle transport equations for electrons and multiple ion species [12]. For the present work, we solve only the equations for $\psi$ (equation (2.11)) and $T_{e}$ (equation (2.12))

$$
\begin{aligned}
& \sigma_{\|}\left(\left.\frac{\partial \psi}{\partial t}\right|_{\hat{\rho}}-\frac{\hat{\rho} \dot{\Phi}_{b}}{2 \Phi_{b}} \frac{\partial \psi}{\partial \hat{\rho}}\right)=\frac{F^{2}}{16 \pi^{2} \mu_{0} \Phi_{b}^{2} \hat{\rho}} \frac{\partial}{\partial \hat{\rho}}\left[\frac{g_{2} g_{3}}{\hat{\rho}} \frac{\partial \psi}{\partial \hat{\rho}}\right] \\
& -\frac{B_{0}}{2 \Phi_{b} \hat{\rho}} V_{\hat{\rho}}^{\prime}\left(j_{\text {aux }}+j_{\mathrm{bs}}\right) .
\end{aligned}
$$




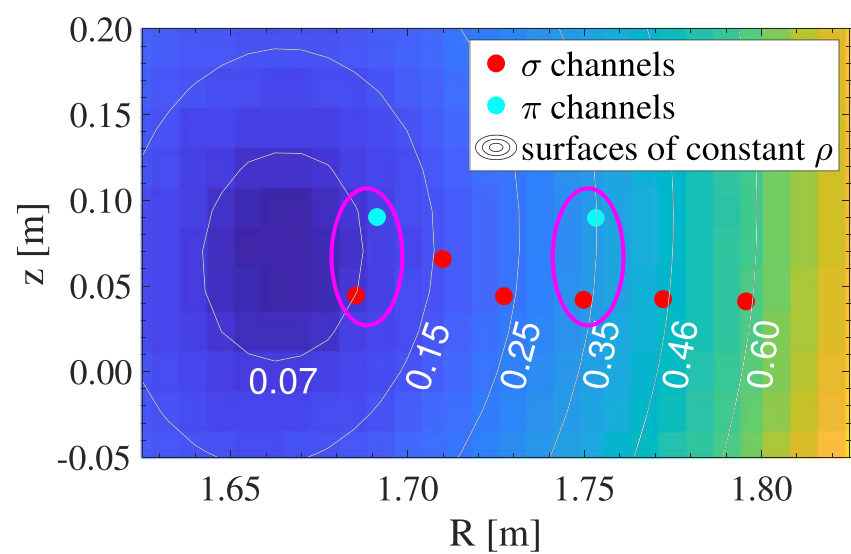

Figure 2. Poloidal plane, CHEASE flux surfaces and corresponding toroidal $\rho$ values. Plotted are the spatial locations of the MSE channels and contour lines of constant $\rho$ intersecting the MSE channels. Note that the values of $\rho$ are subject to change during the shot, shown is the reconstructed grid for $t=3.7 \mathrm{~s}$, shot \#33134. The $q$-profile cannot be constrained by MSE signals in the vicinity of the plasma edge. No MSE channels are available here, however $q_{95}$ is constrained by the total plasma current.

In equation (2.11), $\sigma_{\|}$is the neoclassical conductivity, $j_{\text {aux }}$ is the non-inductive driven current by auxiliary systems, $j_{\mathrm{bs}}$ the bootstrap current, and $g_{2}, g_{3}, F, V_{\hat{\rho}}^{\prime}$ define the geometry of the simulation and are calculated from magnetic equilibria.

The electron energy transport equation is written as:

$$
\begin{gathered}
\frac{3}{2}\left(V_{\hat{\rho}}^{\prime}\right)^{-5 / 3}\left(\left.\frac{\partial}{\partial t}\right|_{\hat{\rho}}-\frac{\dot{\Phi}_{b}}{2 \Phi_{b}} \frac{\partial}{\partial \hat{\rho}} \hat{\rho}\right)\left[\left(V_{\hat{\rho}}^{\prime}\right)^{5 / 3} n_{e} T_{e}\right] \\
+\frac{1}{V_{\hat{\rho}}^{\prime}} \frac{\partial}{\partial \hat{\rho}}\left(-\frac{g_{1}}{V_{\hat{\rho}}^{\prime}} n_{e} \chi_{e} \frac{T_{e}}{\partial \hat{\rho}}+\frac{5}{2} T_{e} \Gamma_{e} g_{0}\right)=P_{e} .
\end{gathered}
$$

With electron temperature, density, thermal diffusivity, convective flux $T_{e}, n_{e}, \chi_{e}, \Gamma_{e}$ and geometric terms $g_{0}, g_{1} . P_{e}$ denotes the sum of the power density sources and sinks. $\Phi_{b}$, $\hat{\rho}=\sqrt{\Phi / \Phi_{\text {edge }}}$, and $B_{0}$ are the toroidal flux enclosed by the LCFS, the normalised square root of the toroidal flux and toroidal magnetic field at the magnetic axis, respectively. The particle flux $\Gamma_{e}$ is neglected in this work.

Equation (2.11) and (2.12) follow the notation of [12], where a detailed description of the individual terms is provided.

The equations are solved on a radial grid corresponding to the normalised toroidal flux and are dependent on the plasma and flux surface shape.

The thermal transport coefficient $\chi_{e}$ is provided by a gradient based empirical transport model [15] with free parameters tuned to match the temperature profiles calculated by the IDE code. The equilibrium calculated by IDE is taken as reference for this analysis.

\subsection{MSE diagnostic}

Equilibrium solvers reconstruct the magnetic equilibrium in the plasma by solving the Grad-Shafranov equation, constrained by various measurements. As a constraint for the current density in the plasma centre, the MSE diagnostic is commonly used. It provides a measure for the local magnetic

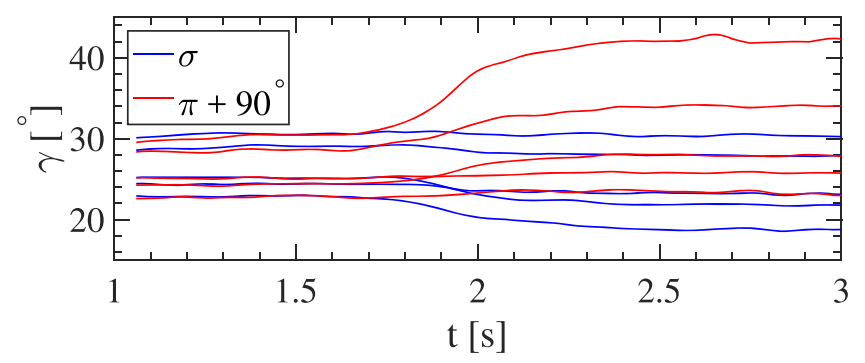

Figure 3. Example of the high-noise contaminated MSE measurements, recorded in AUG shot \#31313. Adapted courtesy of IAEA. Figure from [22]. Copyright 2017 IAEA.

pitch angle $\gamma_{m}$, the angle between $B_{\phi}$ and $B_{\theta}$. The MSE diagnostic measures the light emitted by neutral particles, which are injected into the plasma. Typically, the radiation of the particles injected by the neutral beam injectors is analysed. Due to the Stark effect the emitted light is polarised relative to the magnetic field; measurements of the polarisation angle $\gamma$ can be linked to the pitch angle of the magnetic field projected in a plane perpendicular to the diagnostic's line of sight [4]. The MSE diagnostic at AUG can measure the polarisation angles at 8 different radial positions, with up to two channels per radius. The diagnostic measures the radiation of the Balmer- $\alpha$ line, which, due to the Stark effect, is degenerate into a total of nine lines with different polarisations. The lines can be grouped in two components: $\pi$ - and $\sigma$-lines, which are polarised perpendicular and parallel to the electric field vector. The setup at AUG allows simultaneous measurement of both spectral components at similar radial and vertical positions [20-22].

The configuration of the MSE channels for the shot analysed in this article is shown in figure 2. Six channels of the diagnostic are configured to record the $\sigma$ lines of the Stark spectrum and two for the $\pi$ emission. The measurement location of the $\pi$ channels are in proximity to the $\sigma$ channels. In a shot with high quality MSE data, the relationship $\sigma_{n} \approx \pi_{n}-90^{\circ}$ holds, where $n=1,2$ refers to one of the two channel pairs marked in purple in figure 2 .

A challenge at AUG is the contamination of the recorded signal with background polarised light, originating from reflections on the inner metallic wall of the device. This background polarised light can render the recorded signals unusable in discharges where the line averaged central electron densities exceeds $\left\langle n_{e}\right\rangle \gtrsim 5 \cdot 10^{19}$ [22] (a shot-by-shot analysis is required to determine the quality of the MSE signal). One extreme example of a contaminated measurement is shown in figure 3: the diagnostic was configured to record five $\pi-\sigma$ measurement pairs simultaneously, which should display a $90^{\circ}$ difference. During the shot, this can only be observed within the first $1.7 \mathrm{~s}$, after which the density is increased and the lines begin to diverge [22], rendering the measurement unusable ${ }^{6}$.

6 For completeness we like to mention that similar problems have been reported at Alcator C-Mod, where as a solution the MSE polychromator has been developed [23]. The MSE polychromator measures the $\pi+\sigma$ emission as well as the background polarisation in wavebands close to the measurement wavelengths for every channel. The polarisation angles are then corrected by subtraction of the background. 


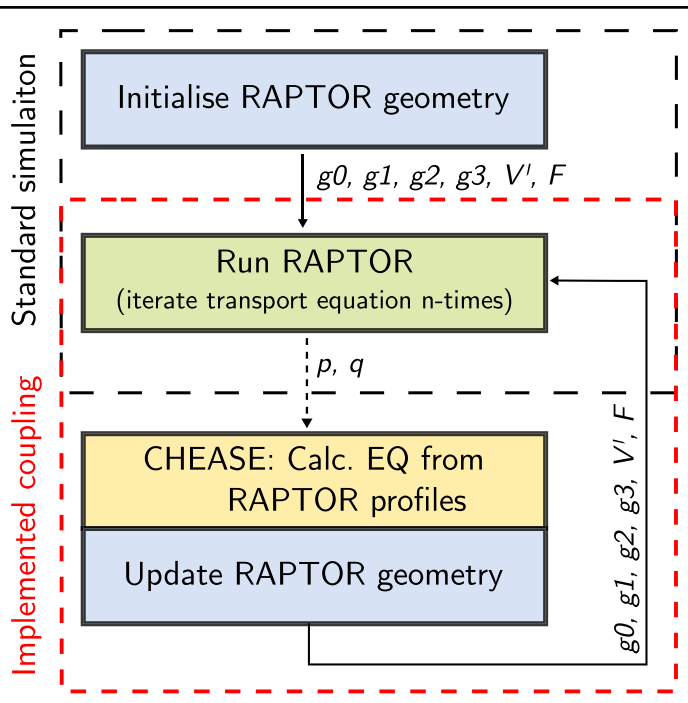

Figure 4. Flow diagram of the implemented coupling between RAPTOR and CHEASE.

The simultaneous measurement of $\pi$ and $\sigma$ lines is used to derive a time-varying estimate of the variance of each MSE measurement: the measurement covariance matrix $R_{k}$, required by the EKF (equation (2.7)), is calculated from the two available $\pi-\sigma$ pairs (see figure 2 ) by calculating their time averaged difference:

$$
\begin{gathered}
\Delta \gamma(t)=\frac{1}{2} \sum_{n=1}^{2} \overline{\left|\left(\gamma_{n}^{\sigma}-\left(\gamma_{n}^{\pi}-90^{\circ}\right)\right)\right|} \\
\equiv \frac{1}{2} \overline{\Delta \Gamma},
\end{gathered}
$$

where the overline denotes that for each time $t, \Delta \Gamma$ is averaged over the previous $20 \mathrm{~ms}$ (the averaging window was chosen to provide statistically relevant results for simulations where the time step is one millisecond, but proved to work well in simulations with a time step of $5 \mathrm{~ms}$ ). The diagonal elements of the covariance matrix, corresponding to the covariances of the individual MSE channels, are then $R_{k}=(\Delta \gamma)^{2}$.

\subsection{Measurement prediction}

The EKF requires the calculations of the predicted measurement from the model. The polarisation angle is calculated by

$$
\gamma=\tan ^{-1}\left(\frac{a_{0} B_{z}+a_{1} B_{r}+a_{2} B_{\phi}}{a_{3} B_{z}+a_{4} B_{r}+a_{5} B_{\phi}}\right),
$$

where the $a_{i}$ are known device specific geometric coefficients, that depend on the setup of the MSE diagnostic. $B_{z}(r, z)$ and $B_{r}(r, z)$ are the vertical and radial components of the poloidal magnetic field $B_{\theta}$, and $B_{\phi}(r, z)$ is the toroidal magnetic field at the measurement location $r, z^{7}$.

\footnotetext{
7 Note that equation (2.15) is a reduced form which neglects contributions from electric fields, which can lead to high uncertainties in advanced plasma scenarios [24]. The radial electric fields cannot be obtained from RAPTOR. For improved accuracy $E_{r}$ can be estimated from ion pressure profiles and toroidal and poloidal velocities [25].
}

From equation (2.15) it is clear that $\gamma$ cannot be derived from the RAPTOR state alone, since RAPTOR calculates 1D profiles and does not reconstruct the $2 \mathrm{D}$ equilibrium. The plasma equilibrium, constrained by RAPTOR's $q$-profile, must be calculated in order to calculate the predicted measurement. Then, by rewriting the poloidal magnetic field as

$$
B_{\theta}=e_{\phi} \times(2 \pi R)^{-1} q^{-1} \nabla \Phi
$$

the polarisation angle can be calculated by using $\Phi=\Phi_{\mathrm{EQ}}$ (i.e. the toroidal flux is obtained from the plasma equilibrium) and $q=q_{\text {RAPTOR}}$. The required coupling between RAPTOR and a equilibrium solver is described next.

\subsection{Coupling between RAPTOR and CHEASE}

The RAPTOR code does not evolve the plasma geometry, but instead assumes it to be fixed throughout the simulation. A time dependent coupling between RAPTOR and an equilibrium reconstruction code is required to calculate the spatial distribution of magnetic fields required to estimate $\gamma$ locally. This requires constraining an equilibrium solver with RAPTOR's $q$-profile and updating the geometry terms in the transport equations from the calculated equilibrium before continuing the iteration of the transport equations. In practice this can be achieved by constraining both codes with the same MSE angles. Since the flux surface shape and $\Phi$ changes slowly compared to $\psi$, and thus $q$, updates of the plasma equilibrium are not required with every iteration of the CDE.

For the presented analysis, RAPTOR has been coupled to CHEASE [17], a fixed-boundary equilibrium solver, which solves the GS equation using specified boundary conditions and internal profiles. The last-closed flux surface and magnetic fields are used as inputs by CHEASE and are directly provided by RAPTOR. The pressure profile in terms of normalised toroidal flux $p(\hat{\rho})$ can also be used as input profile. The use of $\hat{\rho}$ allows a direct coupling with RAPTOR, since it is RAPTOR's radial coordinate, and is less sensitive to changes in the $q$-profiles. This requires CHEASE to transform internally to profiles with respect to the poloidal flux, a solution of the GS equation. Another new option has recently been introduced in CHEASE, namely to provide the $q(\hat{\rho})$ profile as input [26]. In this case CHEASE computes the current density profile $I^{*}$, related to $j_{\phi}$, such as to match the input $q$-profile and iterates until convergence (see equation (50) in [26]). This usually leads to a finite current density at the plasma boundary which can be reduced by slightly modifying the radial derivative of $q$ near the edge. This is sometimes used to study the sensitivity of plasma stability on the edge current density, for example, but it is not needed for this routine since the $I^{*}\left(q_{\text {target }}\right)$ matching performed by CHEASE provides the target $q$-profile without unphysical edge surface currents. Also, CHEASE can use the previous equilibrium as an initial guess which will significantly accelerate the equilibrium calculation.

The implementation allows dynamic updates of the geometry at arbitrary time steps of the simulation; it is illustrated in figure 4 . The implemented coupling calculates the plasma equilibrium based on RAPTOR's $q$ - and pressure 
profiles every $n$th RAPTOR iteration ${ }^{8}$. The equilibrium update frequency is chosen by the user. Too few updates can cause jumps in the calculated polarisation angle, a high update frequency leads to long simulation times. For the presented work it was found sufficient to update the equilibrium every fifth RAPTOR time step.

From the calculated equilibrium, the geometry, i.e. the shape of the poloidal flux surfaces as well as the toroidal flux they enclose (in detail terms $g_{1}, g_{2}, g_{3}, V^{\prime}$ and $F$ in equations (2.11), (2.12) are calculated and updated in RAPTOR before the iteration of the transport equations is continued. This asynchronous implementation allows for a fast and accurate calculation of the polarisation angle, which can be used in offline and real-time simulations alike.

The importance of the equilibrium update is shown figure 5. Here, RAPTOR was run twice with identical simulation parameters, however, in one case the geometry was prescribed and fixed throughout the simulation, and in the second case the geometry terms were updated during the simulation.

The geometry updates do not change the $q$-profile evolution, but result in corrections of $\gamma$ of multiple degrees, essential for a sensitive analysis.

\section{Implementation of the MSE observer}

\subsection{EKF tuning using synthetic data}

The MSE observer is implemented and the performance is evaluated with synthetic data. The aim is to test and validate the implementation and investigate the effect of various settings of the EKF. For this, artificial MSE measurements are generated by simulating an AUG shot with RAPTOR, used in purely predictive mode, as in [15]. Matching plasma equilibria are calculated with CHEASE. From the simulation result and equilibria, the expected polarisation angles are calculated as described in sections 2.4 and 2.5. This simulation and synthetic diagnostic are assumed to correspond to the true evolution of the plasma profiles $\left(q, T_{e}, \ldots\right)$ and corresponding true measurements, i.e. the nominal case.

A normal distributed random noise with standard deviation of $\Delta \gamma=0.5^{\circ}$ is added to the polarisation angles to simulate the effect of measurement noise. This noise level is arbitrarily chosen and approximately five times higher than the acceptable error margins on MSE signals. Furthermore, the model used by the EKF is perturbed with respect to the nominal simulation: firstly, the $q$-profile used to initialise the simulation at $t_{0}=1.5 \mathrm{~s}$ is elevated, see figures $6(\mathrm{~d})$ and 7 at $t_{0}$. Additionally, the electron temperature transport coefficient is reduced by $30 \%$, which yields a higher temperature, higher conductivity and correspondingly different $q$-profile evolution.

\footnotetext{
8 Technically CHEASE was constraint by the parallel current density $j_{\|}$and $p$, which is computational easier and available from RAPTOR. We verified that constraining CHEASE with $j_{\|}$or $q$ results in similar results.
}

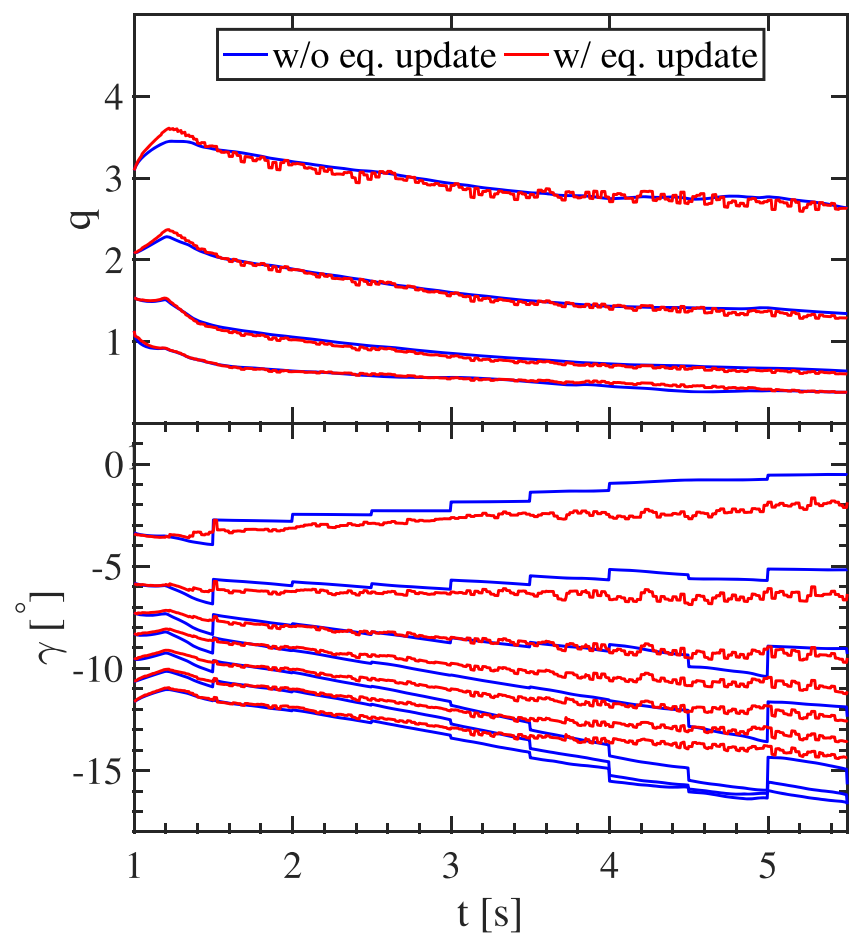

Figure 5. RAPTOR simulations with and without equilibrium update: Top: $q$ values for different $\rho$ values. Bottom: calculated $\gamma$. No simulation parameters were changed resulting in the equal evolution of $q$. The a priori calculated geometry (-) is based on equilibria from IDE which were loaded in half-second intervals. This manifested in step-wise changes in $\gamma$. In the coupled simulation (-) the geometry is updated every 5 th simulation step.

The inputs to the EKF are the perturbed simulation and noisy polarisation angles. The $q$-profile is evaluated to verify that the EKF is able to provide a good estimate of the nominal $q$-profile.

The time evolution of various quantities of the nominal and observer simulation are shown in figure 6. The model mismatch, due to the reduced transport, is clearly visible in the central electron temperature evolution in figure 6(c). For the observer case, the initially perturbed $q$-profile, figure 6(d), converges within $\approx 80 \mathrm{~ms}$ to the $q$-profile of the nominal simulation, only a slight mismatch is visible at the $q$ values towards the plasma centre, which is the most affected by the reduced heat transport.

The innovation sequence $\mathfrak{I}=z_{k}-h\left(\hat{x}_{k \mid k-1}\right)$, the difference between measured and model predicted MSE data, shown in figure 6(e), is expected to be zero mean if the underlying system is linear. For the nonlinear case it still serves as an indication on the performance of the EKF. In the analysed scenario $\mathfrak{I}$ does not converge to a zero mean, but stays close to zero. This implies that a constant correction from the observer on the $q$-profile is required. Contradictory to [19], no disturbance estimation is required due to the relatively large timescales of the current diffusion time.

The $q$-profile of the nominal and perturbed simulation as well as the observer results are compared for various time steps in figure 7. 


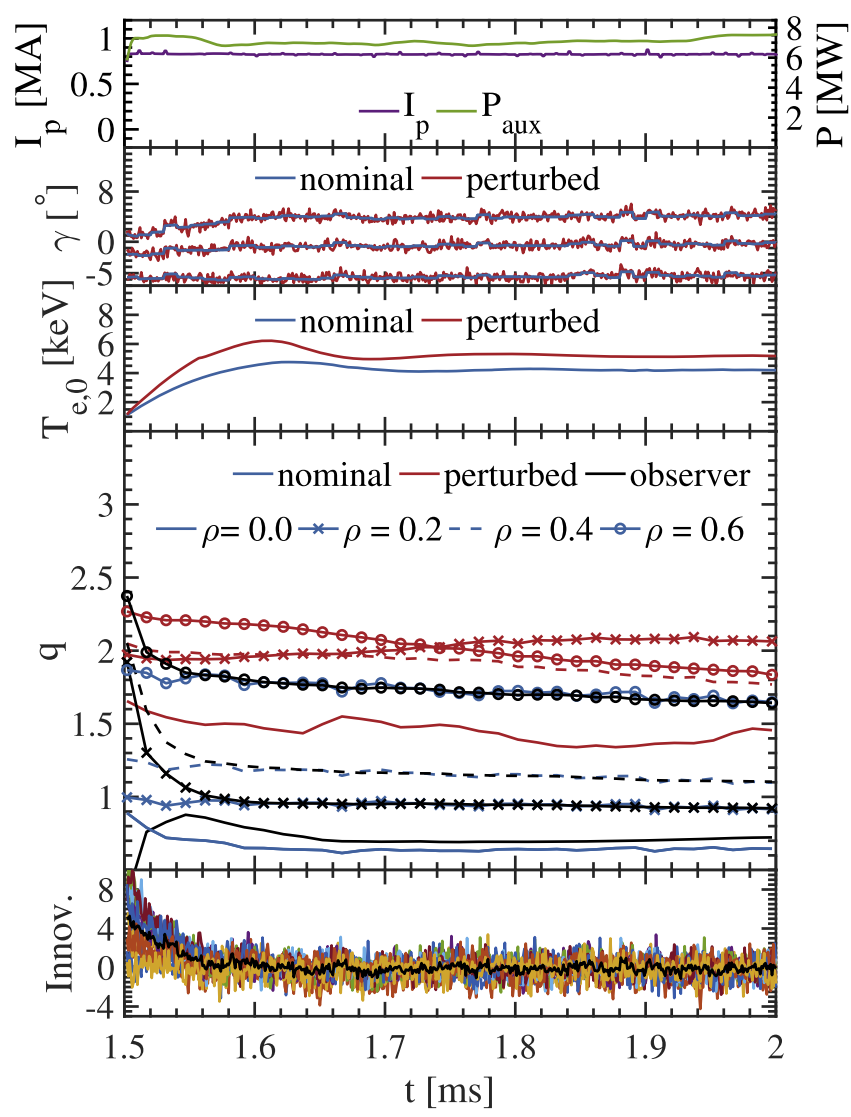

Figure 6. Summary of the MSE observer results using synthetic MSE data (based on AUG shot \#33134). (a) Plasma current and aux. power. (b) Calculated polarisation angle of selected channels without (-) and with $0.5^{\circ}$ noise (-). (c) Central electron temperature of the nominal and perturbed simulation. (d) Comparison of the $q$-value evolution at $\rho=0,0.2,0.4,0.6$ between the nominal simulation, perturbed simulation and MSE observer. (e) Innovation sequence, the difference between measured and model predicted MSE data.

The observer corrects the perturbed $q$-profile to closely approximate the nominal case. The difference in $q$ for each time step between the nominal case and the observer/perturbed case is calculated using the standard deviation figure of merit $[27,28]$

$\sigma(t)=\sqrt{\int_{\rho=0}^{1} \mathrm{~d} \rho\left(q_{\text {obs,pert }}-q_{\mathrm{nom}}\right)^{2}} / \sqrt{\int_{\rho=0}^{1} \mathrm{~d} \rho q_{\mathrm{nom}}^{2}}$.

Averaged over all times $\Delta t>75 \mathrm{~ms}, \quad \bar{\sigma}_{\text {nom } \mid \text { obs }}=0.02$ (between the nominal and observer simulation) is very low compared to $\bar{\sigma}_{\text {nom|pert }}=0.19$ (between the nominal and perturbed case).

The convergence time depends on the choice of the covariance matrix, which is used to tune the EKF. To illustrate the influence of the model covariance, figure 8 shows the innovation sequence for varying $\sigma_{J}$, the value of the diagonal elements of the model covariance matrix $Q_{k}$ (see equation (2.4)). Decreasing covariance results in an increase convergence time. Tuning of the covariance (matrix) is crucial for the operation of the EKF.

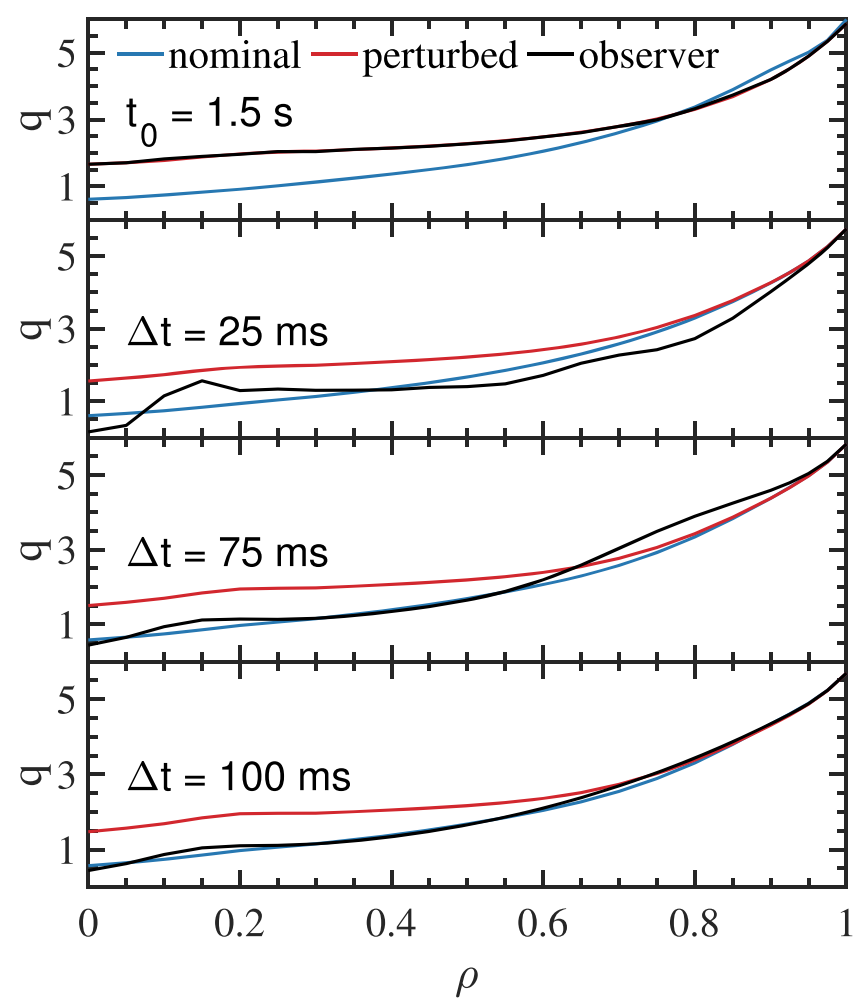

Figure 7. $q$-profile for the reference $(-)$ and perturbed $(-)$ simulation, as well as the $q$-profile resulting from the observer (-). $q$-profiles are plotted for $t_{0}$ and $\Delta t=25,75,100 \mathrm{~ms}$ after the beginning of the simulation. The model of the observer is the perturbed simulation, the input measurements are the noisy polarisation angles.

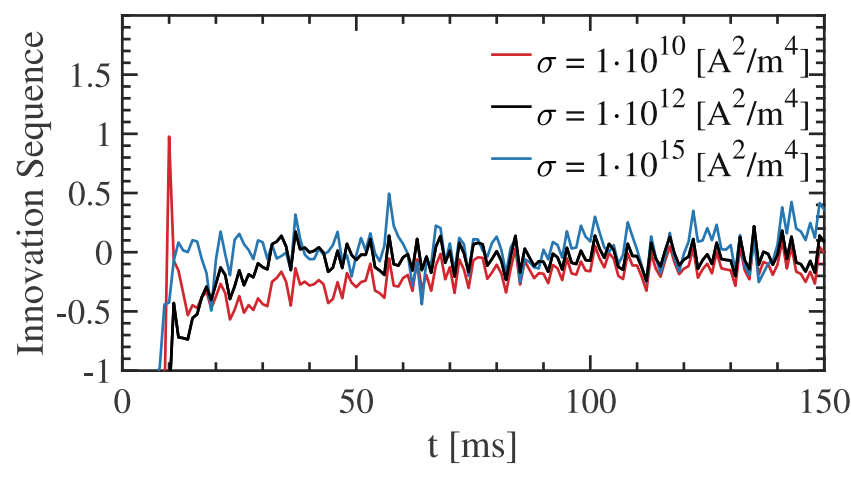

Figure 8. The convergence of the EKF, measured by the innovation sequence $\mathfrak{I}$, can be tuned by the covariance $\sigma_{J}$.

\section{2. q-profile reconstruction of AUG discharge}

After the initial verification using synthetic measurements, the $q$-profile of a full AUG discharge is reconstructed using the MSE observer. A shot with low noise MSE signals is analysed, for which a reference equilibria (reconstructed by MSE constrained IDE [11]) is available. IDE is a GS solver, which, apart from being constrained by the measurements, also evolves the poloidal flux diffusion equation in-between consecutive equilibria reconstructions. The evolved current density profile is used to constrain the next equilibrium solution. The goal of this analysis is for the observer to obtain a 
$q$-profile comparable with the IDE reference. For the analysis, RAPTOR's heat transport model, non-inductive current density deposition profiles from ECRH and NBI as well as the non-inductive driven currents have been tuned to approximate IDE estimates. The density profiles are fixed to match IDE. A comparison between IDE and the RAPTOR simulation without the EKF is shown in figure 9. Here, $q_{\text {edge }}$ matches well due to the constraints at the plasma boundary, however, in the plasma centre, RAPTOR diverges from the reference.

The MSE observer is run with the same RAPTOR configuration used to create figure 9 and the measured polarisation angles of shot \#33134. The goal of the analysis is to obtain a $q$-profile matching the IDE analysis. The safety factor evolution of the MSE observer is shown in figure 10. The observer is active between 1.2 and $7 \mathrm{~s}$, the time during which MSE measurements are available.

The $q$-profile evolution estimated by the MSE observer is in good agreement with the IDE reference. Only in the vicinity of the magnetic axis, where the difference between the pure RAPTOR simulation and IDE is highest, a significant deviation from the IDE reference is notable. At the plasma edge, a match in $q$ between RAPTOR and IDE is guaranteed by the boundary conditions.

The $q$-profiles are compared in figure 11 at four times. The $\mathrm{q}$ values in the vicinity of the magnetic axis differ by $\Delta q \approx 1$ between the RAPTOR simulation without MSE and IDE. The MSE observer corrects the $q$-profile significantly. To compare the correction, the standard deviation figure of merit (see equation (3.1)) is calculated. Since the observer only corrects the $q$-profile in the plasma centre, the integral in equation (3.1) is evaluated like $\int_{\rho=0}^{0.7}$ (for larger values of $\rho$, no MSE channels are available and the observer result matches the RAPTOR simulation without MSE). As it was previously done, the resulting $\sigma(t)$ are averaged over all times. We find a significant improvement from $\bar{\sigma}_{\mathrm{OBS} \mid \mathrm{IDE}}=0.05$ (difference between observer and IDE reference), compared to $\bar{\sigma}_{\mathrm{RAP} \mid \mathrm{IDE}}=0.19$ (disabled observer and IDE reference).

3.2.1. Observer behaviour at signal loss. Lastly, the behaviour of the MSE observer after the loss of the MSE signal is analysed. The previous scenario is repeated, but the MSE input to the observer is cut at $t=5 \mathrm{~s}$. The resulting time evolution of $q$ is shown in figure 12. Immediately after the loss of the measurement signals, the $q$-profile of the observer begins to converge towards the non MSE constrained case.

An advantage of using the MSE observer is the constant availability of polarisation angles which can be passed to a (real-time) Grad-Shafranov solver. In the worst case, i.e. no valid measurements are available, the $q$-profile is predicted solely by the model, whose information of the transport in the plasma can greatly enhance the equilibrium reconstruction. In other words: with the approach described in this article almost any GS solver can easily be constrained by the evolution of the poloidal flux calculated by RAPTOR.

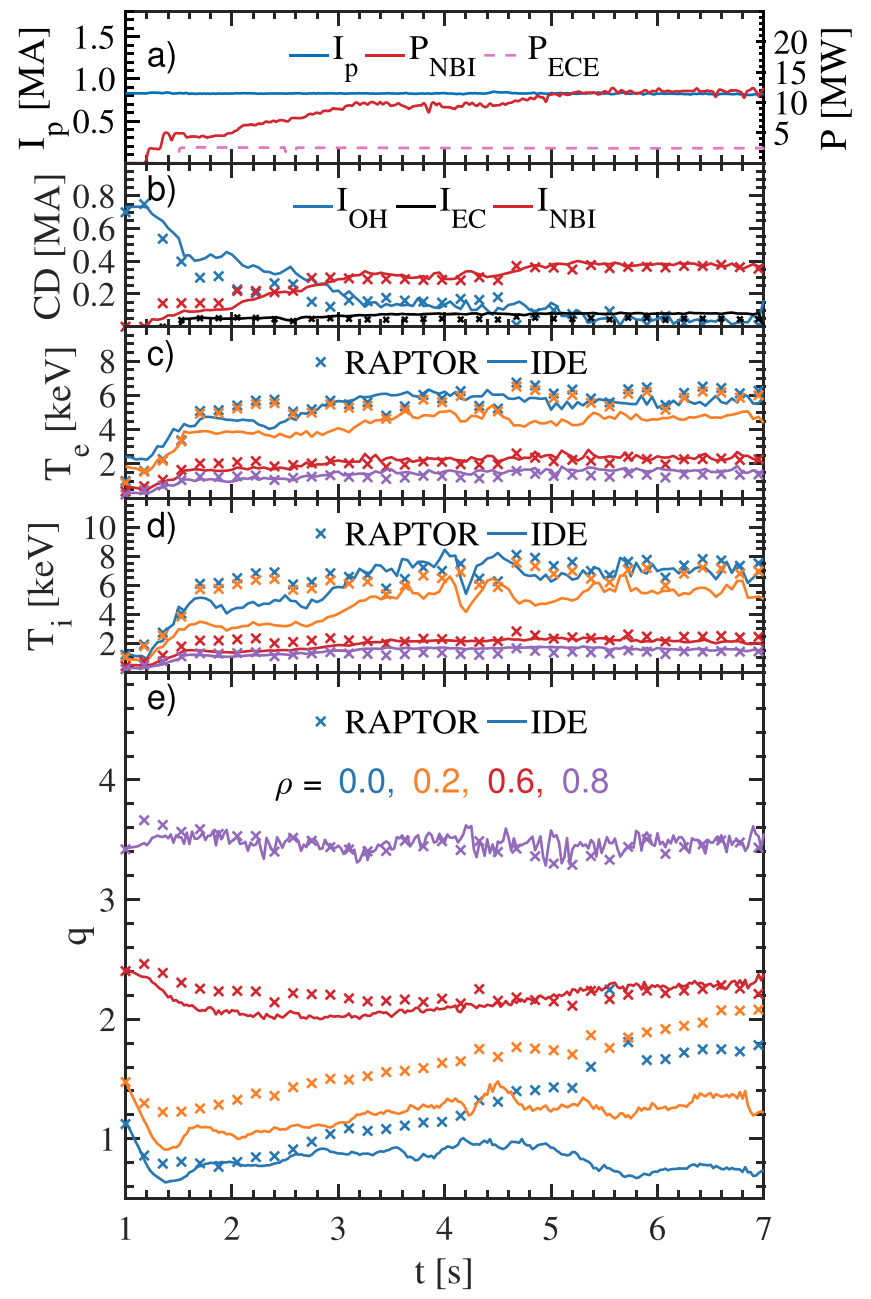

Figure 9. Comparison of IDE analysis and RAPTOR simulation (with deactivated observer) of AUG shot \#33134: (a) Plasma current and aux. power. (b) Current sources from IDE (solid) and RAPTOR (crosses). (c), (d) Electron and ion temperature at different $\rho$. (e) $q$ value evolution at different $\rho$. Temperature and $\mathrm{q}$ values are plotted for $\rho=0,0.2,0.6,0.8$. Note that the shot is not sawtoothing and that due to the high uncertainty of the $q$-profile reconstruction around the magnetic axis, no assessment can be made whether $q_{0}$ drops below one.

\section{Conclusion and outlook}

In this article we have shown that RAPTOR can be constrained by polarisation angle measurements to provide an improved estimation of the $q$-profile. The implemented observer is able to accurately estimate the $q$-profile in simulations where both the measurement and model have been perturbed. Furthermore, it was shown, that in shots where reliable MSE data is available, the MSE observer constrains RAPTOR's $q$-profile to match reference equilibria with minimal offsets. The MSE diagnostic at ASDEX Upgrade suffers from parasitic polarised reflections from the metallic walls, which can render the diagnostic unusable in several scenarios. Due to the setup of the MSE system, which can simultaneously measure $\pi$ and $\sigma$ emission at the same radial location, an (real-time capable) estimate of the measurement 


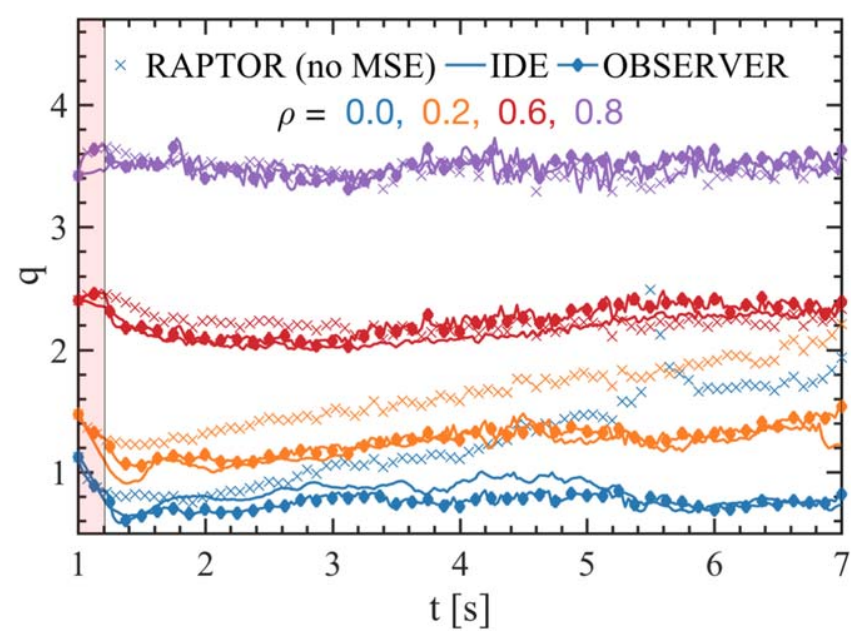

Figure 10. $q$-profile evolution at $\rho=0,0.2,0.6,0.8$. Compared are three cases: RAPTOR without MSE, the reference IDE solution and the observer result. The times where the observer is inactive are shaded in red, here no MSE measurements are available. The RAPTOR time resolution is $5 \mathrm{~ms}$; every 35 th time step is plotted for improved visibility.

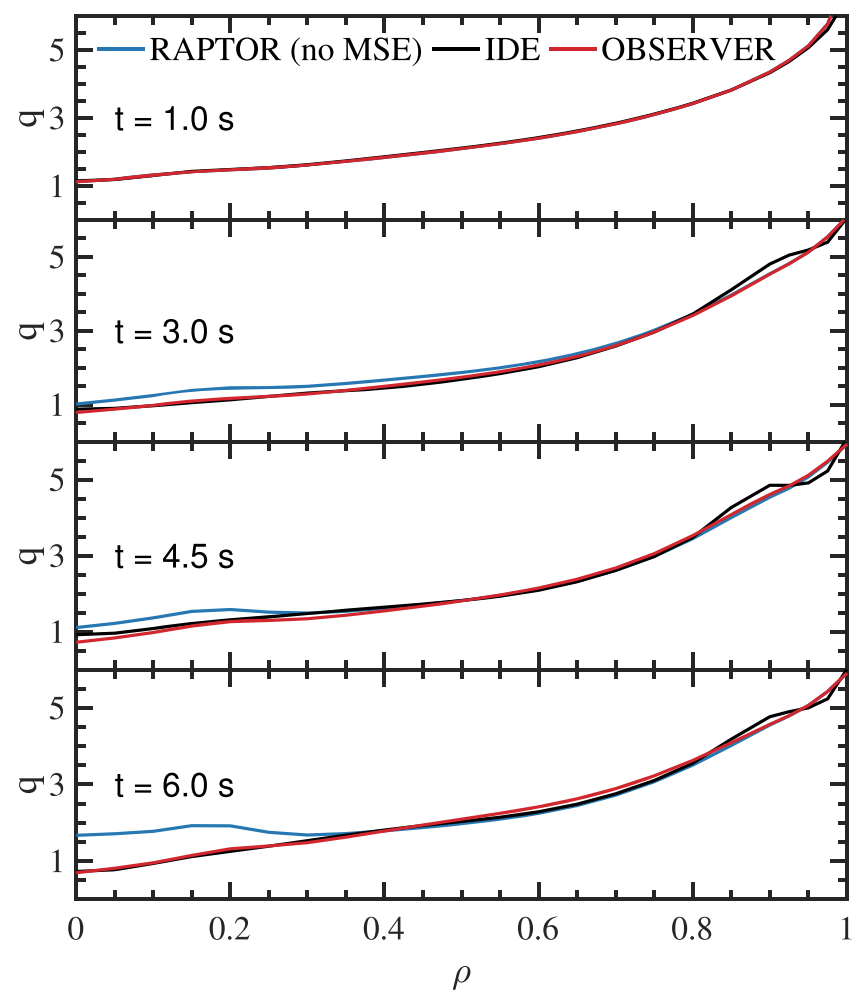

Figure 11. $q$-profile comparison between the nominal simulation $(-)$, IDE (-) and MSE observer (-). Due to the high number of constraints, IDE is able to resolve the plasma edge more accurately than RAPTOR, leading to the divergence of $q$ for $\rho>0.8$.

quality is obtained. With this, the observer continuously updates the covariance matrices and it can provide estimates of the polarisation angle measurements to a GS solver in any discharge scenario, even if physical measurements are unavailable.

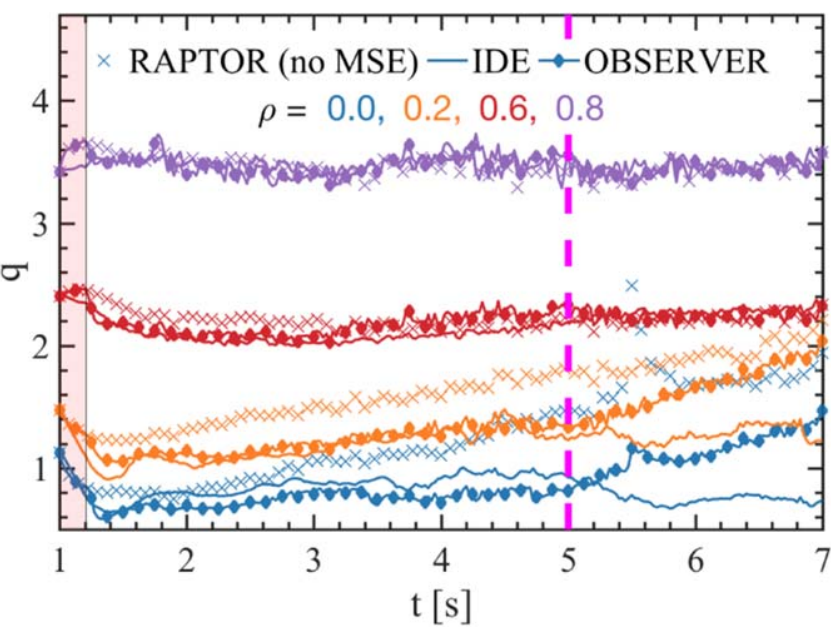

Figure 12. $q$-profile evolution at $\rho=0,0.2,0.6,0.8$. At $t=5 \mathrm{~s}$ the measurement input to the observer is disabled and the simulation continues without being constrained by MSE measurements.

While the work presented here uses the CHEASE equilibrium code, which is a fixed-boundary solver and not capable to run in real-time, this approach can readily be ported to a real-time capable equilibrium code such as LIUQE [29] or JANET [30]. Then, instead of using RAPTOR's $q$-profile, the filtered polarisation angles can be used to constrain the $q$ profile of the equilibrium reconstruction. In principle, this allows any Grad-Shafranov solver to be constrained by the current diffusion, heat and particle transport between consecutive equilibria reconstruction by coupling it to RAPTOR via a virtual MSE diagnostic. The only requirement is that the GS solver can be constrained by MSE measurements.

In the future we plan to implement the observer in AUG's real-time control system, where the RAPTOR code is already implemented, a real-time capable MSE diagnostic is installed and the real-time equilibrium reconstruction code JANET [30] is readily available. For this, the observer must be converted to a Simulink model prior to execution in RT and the corrected MSE signals from the observer passed to JANET, which is already capable of MSE constrained equilibrium reconstruction. Once implemented, a model-based $q$ profile controller can be designed, similar to the already implemented electron temperature controller at ASDEX Upgrade [31], or a plasma profile controller developed for ITER [32]. The RAPTOR code has no constraint on the plasma scenario and can be used to model (and control) monotonic and advanced (e.g. reversed shear) $q$-profiles, using real-time estimates of the driven current from codes such as RT-TORBEAM for electron cyclotron heating $[33,34]$ and RABBIT for NBI [35]. In advanced scenarios, it is important to follow and control the $q$-profile evolution close to the magnetic axis. Here, small uncertainties on the MSE signal can lead to large uncertainties in the $q$-profile reconstruction. The model-based approach can help to gain more accurate insight and control in advanced scenarios. To reduce uncertainties, a central DCN polarimetry channel [36] could possibly be added to the observer, this is however currently not planned. 


\section{Acknowledgments}

This work has been carried out within the framework of the EUROfusion Consortium and has received funding from the Euratom research and training programme 2014-2018 under grant agreement No. 633053. The views and opinions expressed herein do not necessarily reflect those of the European Commission. This work was supported in part by the Swiss National Science Foundation.

\section{ORCID iDs}

M C C Messmer (i) https://orcid.org/0000-0001-8102-0668

F Felici (iD https://orcid.org/0000-0001-7585-376X

\section{References}

[1] Freidberg J P 2007 Plasma Physics and Fusion Energy 1st edn (New York: Cambridge University Press)

[2] Mukhovatov V and Shafranov V D 1971 Plasma equilibrium in a Tokamak Nucl. Fusion 11605

[3] Lao L L, Ferron J R, Groebner R J, Howl W, John H S, Strait E J and Taylor T S 1990 Equilibrium analysis of current profiles in tokamaks Nucl. Fusion 301035

[4] Levinton F M, Fonck R J, Gammel G M, Kaita R, Kugel H W, Powell E T and Roberts D W 1989 Magnetic field pitchangle measurements in the PBX-M tokamak using the motional Stark effect Phys. Rev. Lett. 63 2060-3

[5] Blum J, Boulbe C and Faugeras B 2012 Reconstruction of the equilibrium of the plasma in a Tokamak and identification of the current density profile in real time J. Comput. Phys. 231 960-80

[6] Ko J 2016 Sensitivity of magnetic field-line pitch angle measurements to sawtooth events in tokamaks Rev. Sci. Instrum. 87 11E541

[7] Messmer M C C, Ko J, Chung J, Woo M H, Lee K-D and Jaspers R J E 2018 Evolution of the central safety factor during stabilized sawtooth instabilities at KSTAR Nucl. Fusion 58016030

[8] Hinton F L and Hazeltine R D 1976 Theory of plasma transport in toroidal confinements systems Rev. Mod. Phys. 48 239-308

[9] Felici F, Sauter O, Coda S, Duval B, Goodman T, Moret J-M and Paley J 2011 Real-time physics-model-based simulation of the current density profile in tokamak plasmas Nucl. Fusion 51083052

[10] Fischer R et al (ASDEX Upgrade Team) 2013 Magnetic equilibrium reconstruction using geometric information from temperature measurements at ASDEX Upgrade Proc. 40th EPS Conf. Plasma Phys. ed V Naulin (Mulhouse: European Physical Society) p 2.139

[11] Fischer $\mathrm{R}$ et al 2016 Coupling of the flux diffusion equation with the equilibrium reconstruction at ASDEX Upgrade Fusion Sci. Technol. 69 526-36

[12] Felici F, Citrin J, Teplukhina A A, Redondo J, Bourdelle C, Imbeaux F, Sauter O, Jet Contributors and EUROfusion MST1 Team 2018 Real-time-capable prediction of temperature and density profiles in a tokamak using RAPTOR and a first-principle-based transport model $\mathrm{Nucl}$. Fusion $\mathbf{5 8} 096006$

[13] Kitagawa G and Gersch W 1996 Linear gaussian state space modeling Smoothness Priors Analysis of Time Series (New York: Springer) pp 55-65
[14] Felici F, Sauter O, De Baar M R, Coda S, Duval B, Goodman T, Hommen G, Moret J-M, Voorhoeve R and Steinbuch M 2012 Real-time model-based reconstruction and control of tokamak plasma profiles IAEA Conf. 2012 EX/P3-12

[15] Teplukhina A A, Sauter O, Felici F, Merle A, Kim D, TCV Team, ASDEX Upgrade Team and EUROfusion MST1 Team 2017 Simulation of profile evolution from ramp-up to ramp-down and optimization of tokamak plasma termination with the RAPTOR code Plasma Phys. Control. Fusion 59124004

[16] Van Dongen J, Felici F, Hogeweij G M D, Geelen P and Maljaars E 2014 Numerical optimization of actuator trajectories for ITER hybrid scenario profile evolution Plasma Phys. Control. Fusion 56125008

[17] Lütjens H, Bondeson A and Sauter O 1996 The CHEASE code for toroidal MHD equilibria Comput. Phys. Commun. 97 219-60

[18] Simon D 2006 Optimal State Estimation 1st edn (Hoboken, New Jersey: Wiley) (https://doi.org/10.1002/0470045345)

[19] Felici F, Baar M D and Steinbuch M 2014 A dynamic state observer for real-time reconstruction of the tokamak plasma profile state and disturbances Am. Control Conf. pp 4816-23

[20] Reimer R, Dinklage A, Geiger J, Hobirk J, Reich M, Wolf R and ASDEX Upgrade, and Wendelstein 7-X Teams 2010 Motional Stark effect spectra simulations for Wendelstein 7-X Contrib. Plasma Phys. 50 731-5

[21] Wolf R C, Bock A, Ford O P, Reimer R, Burckhart A, Dinklage A, Hobirk J, Howard J, Reich M and Stober J 2015 Motional Stark Effect measurements of the local magnetic field in high temperature fusion plasmas J. Instrum. 10 P10008

[22] Bock A et al 2017 Non-inductive improved H-mode operation at ASDEX Upgrade Nucl. Fusion 57126041

[23] Mumgaard R T, Scott S D and Khoury M 2016 The multispectral line-polarization MSE system on Alcator C-Mod Rev. Sci. Instrum. 87 87-90

[24] Rice B W, Burrell K H and Lao L L 1997 Effect of plasma radial electric field on motional Stark effect measurements and equilibrium reconstruction Nucl. Fusion 37 517-22

[25] Rice B W, Nilson D G, Burrell K H and Lao L L 1999 Simultaneous measurement of $\mathrm{q}$ and Er profiles using the motional Stark effect in high-performance DIII-D plasmas Rev. Sci. Instrum. 70815

[26] Blondel L and Sauter O 2016 Axisymmetric tokamak equilibria computed with a predefined safety factor profile as CHEASE input, Master project EPFL, Lausanne Report LRP 07/2016 École polytechnique fédérale de Lausanne https://infoscience.epfl.ch/record/261302

[27] Holland C 2016 Validation metrics for turbulent plasma transport Phys. Plasmas 2310

[28] ITER Physics Expert Group on Confinement and Transport 1999 Nucl. Fusion 392175

[29] Moret J M, Duval B P, Le H B, Coda S, Felici F and Reimerdes H 2015 Tokamak equilibrium reconstruction code LIUQE and its real time implementation Fusion Eng. Des. 91 1-15

[30] Giannone L et al (ASDEX Upgrade Team) 2013 A data acquisition system for real-time magnetic equilibrium reconstruction on ASDEX Upgrade and its application to NTM stabilization experiments Fusion Eng. Des. 88 3299-311

[31] Felici F et al 2018 Nucl. Fusion unpublished

[32] Kim S H and Lister J B 2012 A potentially robust plasma profile control approach for ITER using real-time estimation of linearized profile response models Nucl. Fusion 57074002

[33] Reich M, Bilato R, Mszanowski U, Poli E, Rapson C, Stober J, Volpe F, Zille R and ASDEX Upgrade Team 2015 Real-time beam tracing for control of the deposition location of electron cyclotron waves Fusion Eng. Des. 100 73-80 
[34] Poli E et al 2018 TORBEAM 2.0, a paraxial beam tracing code for electron-cyclotron beams in fusion plasmas for extended physics applications Comput. Phys. Commun. 225 36-46

[35] Weiland M, Bilato R, Dux R, Geiger B, Lebschy A, Felici F, Fischer R, Rittich D, Van Zeeland M, The ASDEXUpgrade Team and The Eurofusion MST1 Team 2018 RABBIT:
Real-time simulation of the NBI fast-ion distribution $\mathrm{Nucl}$. Fusion 58082032

[36] Mlynek A, Casali L, Ford O, Eixenberger H and ASDEX Upgrade Team 2014 Fringe jump analysis and implementation of polarimetry on the ASDEX Upgrade DCN interferometer Rev. Sci. Instrum. 85 11D408 